von Schleicher \& Schüll. Als Lösungsmittel verwendeten wir die von Felix und Krekels ${ }^{14}$ angegebene Kombination und außerdem mit 1-proz. Essigsäure ausgeschüttelten Amylalkohol, wie es Grassmann und Hörmann ${ }^{15}$ zur Trennung der Dinitrophenyl-Derivate von Asparagin, Aspara-

$14 \mathrm{~K}$. Felix u. A. Krekels, Hoppe-Seyler's Z. physiol. Chem. 290, 78 [1952]. ginsäure und Glutaminsäure angaben. Als N-endständige Aminosäure wurde Glutaminsäure nachgewiesen, eine andere ließ sich auch bei höheren Konzentrationen nicht auffinden (Abb. 2).

Fräulein E. Grunze danken wir für das Ansetzen der Chromatogramme.

15 W. Grassma n n u. H. H örma n n, HoppeSeyler's Z. physiol. Chem. 292, 24 [1953].

\title{
Bis-cyclopentadienyl $1^{1}$-Verbindungen von Chrom, Molybdän und Wolfram
}

\author{
Von F. A. Cotron ${ }^{2}$ und G. Wilkinson ${ }^{2}$ \\ Chemistry Department, Harvard University, Cambridge, Mass. \\ (Z. Naturforschg. 9 b, 417-418 [1954]; eingegangen am 20. Februar 1954)
}

\begin{abstract}
Die Bis-cyclopentadienyl-Verbindungen des Chroms, Molybdäns und Wolframs wurden nach einer neuen Methode, welche die Reaktion der Metallhalogenide mit CyclopentadienylNatrium in Tetrahydrofuran-Lösung ausnützt, dargestellt.
\end{abstract}

B is - cyclopentadienyl-chrom(II) wurde in sehr kleiner Ausbeute bei thermischer Zersetzung von $\left[\mathrm{Cr}\left(\mathrm{NH}_{3}\right)_{6}\right]\left(\mathrm{C}_{5} \mathrm{H}_{5}\right)_{2}$ erhalten ${ }^{3}$. Jene Verbindung wurde gleichzeitig ${ }^{4}$ in Gramm-Mengen bei der Reaktion zwischen Cyclopentadien und $\mathrm{Cr}(\mathrm{CO})_{6}$ in der Gasphase bei $220-300^{\circ}$ dargestellt.

Wir haben eine neue präparative Methode für Bis-cyclopentadienyl-Verbindungen von großer allgemeiner Verwendbarkeit beschrieben ${ }^{5}$. Diese Methode beruht auf der Reaktion von wasserfreien Halogeniden der Übergangselemente mit Cyclopentadienyl-Natrium in Tetrahydrofuran oder in 1.2-Dimethoxyaethan-Lösung, worin $\mathrm{C}_{5} \mathrm{H}_{5} \mathrm{Na}$ sich mit rot-oranger Farbe löst.

Für die Darstellung von $\left(\mathrm{C}_{5} \mathrm{H}_{5}\right)_{2} \mathrm{Cr}$ werden die Chrom(II)- oder (III)-Halogenide für 1 Stde. mit einer kalten Lösung von $\mathrm{C}_{5} \mathrm{H}_{5} \mathrm{Na}$ in dem Lösungsmittel gerührt. Nach Eindampfen der Mischung wird der Rückstand bei $150^{\circ}$ im Hochvakuum erwärmt, wobei $\mathrm{C}_{10} \mathrm{H}_{10} \mathrm{Cr}$ in einer Ausbeute von etwa $70 \%$ sublimiert. Untersuchungen über das blaue

1 Wir verwenden die Vorsilbe „bis“, um zwischen $\left(\mathrm{C}_{5} \mathrm{H}_{5}\right)_{2} \mathrm{M}$-Verbindungen mit „sandwich“- oder „Doppelkegel“-Struktur und Verbindungen von derselben Formel, aber mit Alkyl-Struktur, z. B. $\left(\mathrm{C}_{5} \mathrm{H}_{5}\right)_{2} \mathrm{Mg}$, zu unterscheiden; die letzteren werden der Nomenklatur von metallorganischen Verbindungen gemäß als Di-cyclopentadienyl-Verbindungen bezeichnet. Wir müssen darauf aufmerksam machen, daß E. O. Fis cher und seine Mitarbb. keine solche Unterscheidung machen und nur die Vorsilbe „di“ benutzen.
Kation ${ }^{4}$, das sich durch die Einwirkung von Säuren auf $\mathrm{C}_{10} \mathrm{H}_{10} \mathrm{Cr}$ erhalten läßt, sind im Gange.

Der eine von uns hat auch die Cyclopentadienylcarbonyle $\mathrm{C}_{5} \mathrm{H}_{5} \mathrm{Mo}(\mathrm{CO})_{5} \mathrm{MoC}_{5} \mathrm{H}_{5}$ und $\mathrm{C}_{5} \mathrm{H}_{5} \mathrm{~W}(\mathrm{CO})_{6} \mathrm{WC}_{5} \mathrm{H}_{5}$, für welche eine 3-SchichtenStruktur mit allen Carbonylgruppen zwischen den Metallatomen vorgeschlagen war, beschrieben ${ }^{4}$. Das diamagnetische, grünliche $\mathrm{C}_{5} \mathrm{H}_{5} \mathrm{Cr}(\mathrm{CO})_{5} \mathrm{CrC}_{5} \mathrm{H}_{5}$ und das fast schwarze $\mathrm{C}_{5} \mathrm{H}_{5} \mathrm{Fe}(\mathrm{CO})_{5} \mathrm{FeC}_{5} \mathrm{H}_{5}$ sind jetzt durch Reaktion von Metallcarbonylen mit Dicyclopentadien in flüssiger Phase erhalten worden. Diese Verbindungen geben infrarote Spektra, die genau denjenigen der Mo- und W-Analogen ähneln, und haben unzweifelhat auch ähnliche Strukturen.

Bis-cyclopentadienyl-Derivate von Mo und W sind jetzt durch die Reaktion von $\mathrm{MoCl}_{5}$ und $\mathrm{WCl}_{6}$ mit $\mathrm{C}_{5} \mathrm{H}_{5} \mathrm{MgBr}$ in $\ddot{A}$ ther und $\mathrm{C}_{5} \mathrm{H}_{5} \mathrm{Na}$ in Tetrahydrofuran oder 1.2- Dimethoxyaethan erhalten worden. $\mathrm{MoCl}_{5}$ wurde in einer Stickstoff-Atmosphäre mit $\mathrm{C}_{5} \mathrm{H}_{5} \mathrm{MgCl}$ in Äther zur Reaktion gebracht. Nach Abdampfen des Lösungsmittels wurde der Rückstand in Stickstoff-Atmosphäre mit 3- $n$. Salzsäure ausgezogen. Die so erhaltene rote Lösung gab mit

2 Zur Zeit im Chem. Lab. A, Technische Hochschule Kopenhagen, Dänemark.

3 E. O. F i s c h e r u. W. H a f n e r, Z. Naturforschg. 8 b, 444 [1953].

4 G. Wilkins on, J. Amer. chem. Soc. 76, 209 [1954].

5 G. Wilkins on u. F. A. Cotton, Chem. and Ind. 307 [1954]. 
Silicowolframsäure und Reineckesäure Niederschläge, nicht aber mit Platinchlorwasserstoffsäure, $\mathrm{H}_{2} \mathrm{PtCl}_{6}$.

$\left.\left[\mathrm{C}_{5} \mathrm{H}_{5}\right)_{2} \mathrm{Mo}(\mathrm{IV}) \mathrm{Cl}\right]\left[\mathrm{Cr}(\mathrm{CNS})_{4}\left(\mathrm{NH}_{3}\right)_{2}\right] \cdot \mathrm{H}_{2} \mathrm{O}$.

Ber. C 28,1; H 3,03; Cl 6,0; Mo 16,0.

Gef. C 29,3; H 3,0; Cl 6,5; Mo 15,9.

Die magnetische Suszeptibilität gibt für diese Verbindung $\mu_{\mathrm{eff}}=3,8 \pm 0,1$ B.M.; die gemessene Suszeptibilität für $\left[\left(\mathrm{C}_{5} \mathrm{H}_{5}\right)_{2} \mathrm{Co}(\mathrm{III})\right]\left[\mathrm{Cr}(\mathrm{CNS})_{4}\left(\mathrm{NH}_{3}\right)_{2}\right]$ $\cdot \mathrm{H}_{2} \mathrm{O}$ gab $\mu_{\text {eff }}=3,9 \pm 0,1$ B.M. Hieraus folgt, daß das $\left[\left(\mathrm{C}_{5} \mathrm{H}_{5}\right)_{2} \mathrm{Mo}(\mathrm{IV}) \mathrm{Cl}\right]^{+}$-Ion diamagnetisch ist.

Durch Extraktion des Grignard-Rückstandes mit 3-5-n. Salpetersäure, oder durch Oxydation der $\mathrm{Mo}(\mathrm{IV})$-Lösung, erhält man eine rotviolette, dichroitische Lösung des $\left[\left(\mathrm{C}_{5} \mathrm{H}_{5}\right)_{2} \mathrm{Mo}(\mathrm{V}) \mathrm{Cl}_{2}\right]^{+}$-Ions, welches gleichfalls Niederschläge mit Silicowolframsäure und Reineckesäure gibt. Diese Lösung gibt außerdem auch einen Niederschlag mit Platinchlorwasserstoffsäure.

$\left[\left(\mathrm{C}_{5} \mathrm{H}_{5}\right)_{2} \mathrm{Mo}(\mathrm{V}) \mathrm{Cl}_{2}\right]_{2}\left[\mathrm{PtCl}_{6}\right]$.

Ber. C 24,0; H 2,0; Cl 35,0; Pt 19,5; Mo 19,0.

Gef. C 23,7; H 2,2; Cl 36,5; Pt 19,0; Mo 19;23.

Die Verbindung ist paramagnetisch mit $\mu_{\text {eff }}=1,5$ $\pm 0,1$ B.M., was auf das Vorhandensein eines einzigen ungepaarten Elektrons deutet. [1952].

6a G. Wilkinson, J. Amer. chem. Soc. 74, 6146
$\mathrm{MoCl}_{5}$ wurde auch unter Stickstoff mit $\mathrm{C}_{5} \mathrm{H}_{5} \mathrm{Na}$ in Tetrahydrofuran gerührt. Nach 2 Stdn. wurde das Lösungsmittel abdestilliert und der Rückstand mit Chloroform ausgezogen. Kristallisation aus Chloroform ergab grüne Nadeln von $\mathrm{C}_{10} \mathrm{H}_{10} \mathrm{Mo}(\mathrm{IV}) \mathrm{Cl}_{2}$.

Ber. C 40,3; H 3,63; Mo 32,2; Cl 23,8.

Gef. C 40,1; H 3,6; Mo 32,15; Cl 23,75.

Weiter wurde gefunden, daß W-hexachlorid ganz analog reagiert; wir haben z. B. das Chloroplatinat isoliert.

$$
\left[\left(\mathrm{C}_{5} \mathrm{H}_{5}\right)_{2} \mathrm{~W}(\mathrm{~V}) \mathrm{Cl}_{2}\right]_{2}\left[\mathrm{PtCl}_{6}\right] \text {. }
$$

Ber. C 20,4; H 1,72; Cl 30,1; Pt 16,6; W 31,2.

Gef. C 20,3; H 1,80; Cl 30,3; Pt 16,6; W 31,2.

Alle die hier besprochenen Mo- und W-Verbindungen geben infrarote Absorptions-Spektra, die typisch für die Bis-cyclopentadienyl-Verbindungen $\operatorname{sind}^{6}$. Sie zeigen bei $3,27 \mu$ eine einzelne $\mathrm{C}-\mathrm{H}$-Bande und weitere Banden bei $\sim 7,0, \sim 9,0, \sim 9,4, \sim 10,0$ und $\sim 11,8 \mu$; die Messungen wurden in Alkali-Halogenid-Druckplatten vorgenommen. Weitere Mitteilungen über diese Arbeit und über Chrom- und Eisen-cyclopentadienylcarbonyle werden demnächst erscheinen.

${ }^{6 b}$ E. R. Lippincott u. R. D. Nelson, J. Chem Phys. 21, 1307 [1953].

\title{
Über die Stabilität der geraden Alkohole, Mercaptane und Alkylbenzole bei Elektronenstoß
}

\author{
Von M. PAHL \\ Aus der Forschungsstelle für Spektroskopie in der Max-Planck-Gesellschaft, Hechingen \\ (Z. Naturforschg. 9 b, 418-428 [1954]; eingegangen am 6. Mai 1954) \\ Herrn Professor H.Schüler zum 60. Geburtstag gewidmet
}

\begin{abstract}
Aus den Angaben des "Catalog of Mass Spectral Data" wird empirisch eine quantitative Beziehung zwischen der Stabilität der Mutterionen $\left(W_{\mathrm{MI}}\right)$ und der Anzahl der BindungsElektronen $\zeta$ des Alkylrestes gefunden. Sie lautet im Falle der S-Alkohole $W_{\mathrm{M}} \cdot \zeta=$ const und hat bei den Alkylbenzolen dieselbe Form, wenn hierbei $W_{\mathrm{M}}$ nur auf den Zerfall des Alkylrestes ohne die Ringaufspaltung bezogen wird. Bei den Alkanen und O-Alkoholen nimmt $W_{\mathrm{M}}$ rascher $\mathrm{ab}$, etwa mit $W_{\mathrm{M}} \cdot \zeta^{2}=$ const'. Die Stabilität $W_{\mathrm{M}}$ hängt nicht mit der Form der Massenspektren zusammen, sondern ist offenbar bestimmt durch einen Prozeß, der innerhalb $\leqq 10^{-14}$ sec das gesamte System der Bindungs-Elektronen im Molekül erfaßt.
\end{abstract}

\section{1.}

$U_{\mathrm{l}}^{\mathrm{b}}$ ber die Stabilität organischer Moleküle gegen Elektronenstoß wurde in einer kürzlich veröffentlichten Arbeit ${ }^{1}$ ein quantitativer Überblick ge-

1 M. P a h l, Z. Naturforschg. 9b, 188 [1954]. geben, der aus den bis zum 30. Juni 1952 erschienenen Daten des „Catalog of Mass Spectral Data “2 errechnet war. In dieser Zusammenstellung fiel besonders

2 Catalog of Mass Spectral Data des American Petroleum Institute Research Project 44, Carnegie Institute of Technology, Pittsburgh, Pennsylvania. 